\title{
Podocalyxin enhances breast tumor growth and metastasis and is a target for monoclonal antibody therapy
}

Kimberly A Snyder ${ }^{1 \dagger}$, Michael R Hughes ${ }^{1 \dagger}$, Bradley Hedberg${ }^{2}$, Jill Brandon², Diana Canals Hernaez ${ }^{1}$, Peter Bergqvist ${ }^{2}$, Frederic Cruz², Kelvin Po ${ }^{2}$, Marcia L Graves ${ }^{3}$, Michelle E Turvey ${ }^{4}$, Julie S Nielsen ${ }^{1}$, John A Wilkins ${ }^{5}$, Shaun R McColl, John S Babcook ${ }^{2}$, Calvin D Roskelley ${ }^{3}$ and Kelly M McNagny ${ }^{1^{*}}$

\begin{abstract}
Introduction: Podocalyxin (gene name PODXL) is a CD34-related sialomucin implicated in the regulation of cell adhesion, migration and polarity. Upregulated expression of podocalyxin is linked to poor patient survival in epithelial cancers. However, it is not known if podocalyxin has a functional role in tumor progression.

Methods: We silenced podocalyxin expression in the aggressive basal-like human (MDA-MB-231) and mouse (4T1) breast cancer cell lines and also overexpressed podocalyxin in the more benign human breast cancer cell line, MCF7. We evaluated how podocalyxin affects tumorsphere formation in vitro and compared the ability of podocalyxin-deficient and podocalyxin-replete cell lines to form tumors and metastasize using xenogenic or syngeneic transplant models in mice. Finally, in an effort to develop therapeutic treatments for systemic cancers, we generated a series of antihuman podocalyxin antibodies and screened these for their ability to inhibit tumor progression in xenografted mice.

Results: Although deletion of podocalyxin does not alter gross cell morphology and growth under standard (adherent) culture conditions, expression of PODXL is required for efficient formation of tumorspheres in vitro. Correspondingly, silencing podocalyxin resulted in attenuated primary tumor growth and invasiveness in mice and severely impaired the formation of distant metastases. Likewise, in competitive tumor engraftment assays where we injected a 50:50 mixture of control and shPODXL (short-hairpin RNA targeting PODXL)-expressing cells, we found that podocalyxin-deficient cells exhibited a striking decrease in the ability to form clonal tumors in the lung, liver and bone marrow. Finally, to validate podocalyxin as a viable target for immunotherapy, we screened a series of novel antihuman podocalyxin antibodies for their ability to inhibit tumor progression in vivo. One of these antibodies, PODOC1, potently blocked tumor growth and metastasis.
\end{abstract}

Conclusions: We show that podocalyxin plays a key role in the formation of primary tumors and distant tumor metastasis. In addition, we validate podocalyxin as potential target for monoclonal antibody therapy to inhibit primary tumor growth and systemic dissemination.

\footnotetext{
* Correspondence: kelly@brc.ubc.ca

'Equal contributors

${ }^{1}$ The Biomedical Research Centre, University of British Columbia, 2222 Health

Sciences Mall, Vancouver, BC V6T 1Z3, Canada

Full list of author information is available at the end of the article
} 


\section{Introduction}

Although most human cancers begin as primary focal lesions, metastasis of these primary tumors to distant sites heralds advanced stage disease, poor prognosis and eventual patient death [1]. For this reason, biomarkers that identify tumors likely to metastasize, and the generation of therapeutics that can inhibit metastasis, are key to improving patient survival. Although adjuvant therapies have been developed for several types of breast tumors, triple-negative breast cancers (estrogen receptor (ER)-, progesterone receptor (PgR)- and human epidermal growth factor receptor 2 (HER2)negative) are particularly challenging to treat because of their highly aggressive nature and a lack of welldefined therapeutic targets on these cells [2].

Podocalyxin (also known as PCLP1, MEP21, gp135, TRA-1-60, TRA-1-81 and GCTM2) is a CD34-related sialomucin and a well-known marker of embryonic stem cells, embryonal carcinomas, neoplastic hematopoietic cells [3-6] and a variety of normal cells during embryonic development, where it plays a key role in tissue morphogenesis [7-9]. We previously showed that podocalyxin (gene name $P O D X L)$ is upregulated on a subset of primary breast tumors and is an independent predictor of progression, metastasis and poor outcome [10]. Subsequent studies have confirmed podocalyxin as a prognostic indicator of poor outcome in a variety of malignancies, including ovarian, prostate, renal, pancreatic, thyroid, glioblastoma, astrocytoma, colorectal and bladder cancers [10-18]. Ectopic expression of PODXL enhances tumor aggressiveness in vitro. MCF7 breast tumor cells engineered to express high levels of murine podocalyxin $\left(M C F 7^{\text {Podxl }}\right.$ ) exhibit increased migration in vitro, altered morphogenesis and disrupted cell-cell and cell-matrix contacts $[10,19,20]$. In addition, podocalyxin has been shown to play a role in the control of cell migration and the expression of matrix metalloproteinases MMP1 and MMP9 [17,21]. Collectively, these studies establish a correlation between podocalyxin expression, tumor aggressiveness and poor outcome (reviewed by McNagny et al. [22]). However, the functional significance of podocalyxin expression by primary tumors and its influence on metastatic progression in vivo have yet to be thoroughly evaluated. In the present study, we have addressed this issue by silencing podocalyxin expression in the highly aggressive triple-negative basal-like human breast cancer cell line, MDA-MB-231, or overexpressing it in a well-differentiated, ER-positive and PgR-positive, luminal-like human breast cancer cell line, MCF7 [23]. We found that podocalyxin is required for efficient tumorsphere formation in both MCF7 and MDA-MB-231 cells. Moreover, suppression of PODXL in MDA-MB-231 cells profoundly impairs formation of primary tumors and secondary metastasis in xenografted mice. We recapitulated this finding in an immunocompetent mouse tumor model by silencing podocalyxin expression in 4T1 cells (a mouse mammary tumor line) and engrafting these cells in syngeneic BALB/c mice. Finally, we developed a novel podocalyxin-specific monoclonal antibody $(\mathrm{mAb})$ that delays xenografted tumor formation and metastatic disease in mice. These data validate podocalyxin as a regulator of tumor progression and a novel therapeutic target.

\section{Methods}

\section{Cell culture}

MDA-MB-231, MCF7 and 4T1 cells (American Type Culture Collection, Manassas, VA, USA) were grown as monolayers on tissue culture-treated plastic plates. All cell lines were maintained in low passage $(<15)$. Both MDA-MB-231 and MCF7 human breast tumor cell lines were cultured in Dulbecco's modified Eagle's medium (DMEM) supplemented with $10 \%$ fetal bovine serum (FBS), penicillin and streptomycin. 4T1 BALB/c mouse-derived mammary tumor cells were cultured in DMEM supplemented with $10 \%$ FBS, $2 \mathrm{mM}$ glutamine, nonessential amino acids, penicillin and streptomycin. All cell lines were cultured in a humidified 5\% $\mathrm{CO}_{2}$ incubator at $37^{\circ} \mathrm{C}$.

\section{Transduction}

MDA-MB-231 cells were labeled with green fluorescent protein (GFP) or red fluorescent protein (RFP) using retroviral vectors pLNCX2-GFP or pLNCX2-RFP, respectively (Clontech Laboratories, Mountain View, CA, USA). Human PODXL was silenced in MDA-MB-231 cells by lentiviral infection using pLKO.1 containing either a scrambled short-hairpin RNA (shRNA) (shCTRL) or a PODXL-targeting shRNA (RHS3979-9848792, shPODXL) as recommended by the manufacturer (Dharmacon, Lafayette, CO, USA). All cell lines were derived from pooled cultures of infected cells. Cells were cultured under continuous drug selection with puromycin $(4 \mu \mathrm{g} / \mathrm{ml}$; Invitrogen, Carlsbad, CA, USA) and G418 (1 mg/ml; Calbiochem, San Diego, CA, USA). PODXL-transfected MCF7 cells were described previously $[10,19]$. Cells were cultured under continuous selection with gentamicin $(50 \mu \mathrm{g} / \mathrm{ml}$; Calbiochem).

Predicted shRNA sequences to target murine 4T1 Podxl were identified using pSicoOligomaker v1.5 freeware (http:// web.mit.edu/jacks-lab/protocols/pSico.html). Three individual shRNA oligomers were each cloned into the HpaI and XhoI sites of the pLL3.7 lentiviral vector. Firefly luciferaseexpressing 4T1 (4T1-luc) cells were maintained under selection in G418 (400 $\mu \mathrm{g} / \mathrm{ml}$; Calbiochem). To produce lentiviral particles, 293T cells were cotransfected with $10 \mu \mathrm{g}$ of pLL3.7 and the appropriate packaging plasmids $(3.5 \mu \mathrm{g}$ of pVSVg, $3.5 \mu \mathrm{g}$ of pRSV-Rev, $6.5 \mu \mathrm{g}$ of $\mathrm{pMDLgag} / \mathrm{pol}$ ) by calcium phosphate transfection. Lentivirus-containing media were collected 36 hours post-transfection and transferred to 
subconfluent $4 \mathrm{~T} 1$ cells seeded 1 day earlier. The viruscontaining medium was replaced with regular growth media after 48 hours and incubated for an additional 48 hours. The cells were then harvested for analysis of expression of mouse podocalyxin RNA and protein. 4T1 cells with the most efficient knockdown were used for all studies and cultured with gentamicin (50 $\mu \mathrm{g} / \mathrm{ml}$; Calbiochem).

\section{Quantitative RT-PCR}

RNA isolation was performed using TRIzol reagent (Life Technologies, Carlsbad, CA, USA) according to the manufacturer's instructions. Total RNA $(2 \mu \mathrm{g})$ was reverse-transcribed using a high-capacity cDNA reverse transcription kit (Life Technologies). Real-time quantitative PCR was performed using a SYBR FAST qPCR kit (Kapa Biosystems, Wilmington, MA, USA). The PODXL-specific primers used were 5'-CTCACCGGGGACTACAACC3' (forward) and 5'-GCCTCCTCTAGCCACGGTA-3' (reverse). Relative expression of $P O D X L$ was determined relative to GAPDH in each reaction.

\section{Tumorsphere assay}

MDA-MB-231 and MCF7 cells were harvested, and spheres were cultured in MammoCult ${ }^{\mathrm{Tu}}$ medium (StemCell Technologies, Vancouver, BC, Canada). After 7 days, tumorspheres larger than $60 \mu \mathrm{m}$ in diameter were counted manually using a counting grid. Tumorsphere-forming efficiency was calculated as follows: number of tumorspheres divided by number of cells initially plated times 100 .

\section{In vivo tumor growth and lung metastasis}

For in vivo experiments, we used 6- to 12-week-old female nonobese diabetic severe combined immunodeficiency, interleukin 2 receptor gamma chain deficient, NOD.Cg-Prkdc $c^{\text {scid }} I l 2 r g^{t \mathrm{~m} 1 \mathrm{Wjl} /} / \mathrm{SzJ}$ (NSG) mice or BALB/cJ mice (The Jackson Laboratory, Bar Harbor, ME, USA). Animals were maintained in a specific pathogen-free facility at the University of British Columbia (UBC) Biomedical Research Centre. All experiments were conducted with approval of the UBC Animal Care Committee.

Primary tumor development was examined following subcutaneous (s.c.) injection of MDA-MB-231 cells $(1 \times$ $10^{6}$ ) prepared in BD Matrigel ${ }^{\mathrm{TN}}$ (BD Biosciences, San Jose, CA, USA) into the right hind flank of NSG mice. Tumor growth was measured using manual calipers, and the tumor volume was estimated using the following formula: length times width ${ }^{2}$ divided by 2 . Final tumor masses were measured after excision and the tumors were retained for histochemical analyses. Flow cytometry was performed on lung digests to enumerate tumor cells based on detection of GFP or RFP fluorescence.

\section{Competitive experimental metastases}

To examine experimental metastasis, a 50:50 mixture of 0.5 to $2.0 \times 10^{5}$ shCTRL $^{\text {RFP }}$ (or shCTRL ${ }^{\text {GFP }}$ ) and shPODXL ${ }^{\text {GFP }}$ (or shPODXL ${ }^{\mathrm{RFP}}$ ) MDA-MB-231 cells were resuspended in $100 \mu \mathrm{l}$ of Hanks' balanced salt solution and injected into the tail vein of NSG mice. At day 3, 7 or 14 postinjection, mice were killed using 2,2,2-tribromoethanol (Avertin; Sigma-Aldrich, St Louis, MO, USA), then perfused through the right ventricle with $10 \mathrm{ml}$ of phosphate-buffered saline (PBS) containing $2 \mathrm{mM}$ ethylenediaminetetraacetic acid (EDTA), and the lungs (and, in some experiments, liver, femurs and tibias) were removed. Lungs were digested in collagenase/dispase solution as described elsewhere [24], and GFP-positive or RFP-positive tumor cells were detected by flow cytometry. At 6 weeks postinjection, NSG mice were killed and perfused as described above, but tumor nodules on the surface of lungs and livers were manually counted using a Leica Fluo ${ }^{\mathrm{Tm}}$ dissecting microscope (Leica Microsystems, Buffalo Grove, IL, USA) and QImaging ${ }^{\mathrm{Tm}}$ software (QImaging, Surrey, BC, Canada). In addition, lung, liver and bone marrow cells were prepared as described previously and analyzed by flow cytometry.

\section{Flow cytometry}

Staining was performed with PBS containing 2\% FBS, 2 mM EDTA and $0.05 \%$ sodium azide. MDA-MB-231 cells were stained with a primary antibody $(\mathrm{Ab})$ against podocalyxin (goat antihuman podocalyxin antibody (antiPODO Ab); R\&D Systems, Minneapolis, MN, USA) or a goat immunoglobulin G (IgG) isotype control (Iso) and followed with a chicken anti-goat Alexa Fluor (AF) 647coupled secondary Ab (Molecular Probes, Eugene, OR, USA) for 30 minutes at $4^{\circ} \mathrm{C}$ and analyzed using a $\mathrm{BD}$ LSR II flow cytometer (BD Biosciences). Murine 4T1-luc cells were labeled with allophycocyanin-conjugated rat anti-mouse podocalyxin Ab (R\&D Systems) and analyzed by flow cytometry. Rat IgG2b was used as an isotype control.

\section{Experimental lung metastasis}

A total of $1 \times 10^{5}$ vector control (VC) or shPODXL 4T1luc cells were injected intravenously (i.v.) into the lateral tail vein $B A L B / c$ mice. Lungs were perfused and excised as described above. Tumor burden was assessed by counting nodules visible on the surface of the lungs using a dissecting microscope and then corroborated by performing a luciferase assay of homogenized lung tissue.

\section{Luciferase enzymatic assay}

Total luciferase activity was assayed from lungs harvested from BALB/c mice injected i.v. with 4T1-luc cells. Lungs were homogenized in cell lysis buffer (Promega, Madison, WI, USA). Protein concentration was determined using a Thermo Scientific Pierce bicinchoninic acid protein assay kit (Pierce Biotechnology, Rockford, IL, USA). The Dual- 
Luciferase Reporter Assay System (Promega) was used to detect luciferase activity. In these experiments, $20 \mu \mathrm{l}$ of sample supernatant was mixed with $50 \mu \mathrm{l}$ of luciferase assay reagent, and luciferase activity was quantified using a SpectraMax L microplate reader (Molecular Devices, Sunnyvale, CA, USA). The results are reported as relative light units.

\section{Therapeutic antibody production}

New Zealand White rabbits were immunized with A-172 glioblastoma cells that express high levels of tumorglycosylated human podocalyxin on their cell surface. Rabbit mAbs were rescued as previously described [25]. Briefly, individual B-cell clones were isolated from animals whose sera recognized MDA-MB-231 cell-expressed podocalyxin extracellular domain by enzyme-linked immunosorbent assay. Next, supernatants were screened against MDA-MB231 and human embryonic kidney 293 (HEK293) cells with and without podocalyxin on their surface (both cell lines express endogenous podocalyxin) to ensure immunoreactivity to the native protein and minimal nonspecific binding to PODXL-deficient cells. Finally, supernatants were also screened using Chinese hamster ovary $(\mathrm{CHO})$ cells expressing podocalyxin and CD34 to ensure podocalyxin specificity. By comparing binding selectivity for podocalyxin expressed on tumor and normal cells, B-cell clones that produced Abs with favorable binding profiles to tumor cells were selected for cloning, scale-up production and in vivo screening.

\section{Preclinical mouse model to assess anti-podocalyxin therapeutic antibody efficacy}

Candidate anti-PODO Abs were selected based on the level of binding to known podocalyxin-expressing tumor cell lines (Table 1$)$. MDA-MB-231 ${ }^{\mathrm{RFP}}$ tumor cells $\left(1 \times 10^{6}\right.$ cells $)$ were incubated with $25 \mu \mathrm{g}$ of anti-PODO Ab (PODOC1 through PODOC8) or Iso $\mathrm{Ab}$ (anti-ovalbumin) at room temperature for 30 minutes. Prior to injection, the tumor cell/Ab mixture was diluted in Matrigel ${ }^{\mathrm{Tn}}$ and injected s.c. into the flank region of NSG mice. For systemic therapy, beginning on day 14 after tumor injection, mice were administered $100 \mu \mathrm{g}$ of $\mathrm{Ab}(4.5 \mathrm{mg} / \mathrm{kg})$ by intraperitoneal (i.p.) injection twice weekly. Tumor dimensions were measured every 3 days until the mice were killed on day 27 .

\section{Histological analysis}

Formalin-fixed, paraffin-embedded tumor specimens were serially sectioned. Representative sections were deparaffinized and stained with hematoxylin and eosin (H\&E) or Ki$67 \mathrm{Ab}$ (1:700; Thermo Scientific, Waltham, MA, USA) followed by donkey anti-rabbit AF488 secondary Ab (1:1,000; Invitrogen). ProLong Gold Antifade mounting compound with 4',6-diamidino-2-phenylindole dihydrochloride (DAPI) nuclear stain (Life Technologies) was used to mount slides. H\&E-stained sections were examined qualitatively for evidence of muscular invasion and tumor border integrity.

\section{Statistical analysis}

Data are expressed as the mean \pm standard error of the mean (SEM) unless indicated otherwise. A Student's $t$-test was conducted for evaluation of statistical significance. Data generated from time-dependent studies were analyzed by two-way analysis of variance. $P<0.05$ was considered to be statistically significant. All data presented in the figures are representative of at least two independent experiments.

\section{Results}

\section{Podocalyxin promotes tumorsphere formation in vitro}

To examine the role of podocalyxin in tumor progression, we silenced expression in MDA-MB-231 human breast cancer cells using a shRNA-containing lentivirus (shPODXL). RFP- and GFP-labeled MDA-MB-231 cells were used as a method for subsequent tracking of knockdown cells in vivo. Gene expression analyses confirmed attenuated PODXL expression in shPODXL cells (Figure 1A). In addition, flow cytometric analyses confirmed that cell surface podocalyxin expression is efficiently reduced in shPODXL ${ }^{\text {GFP }}$ cells (threefold decrease) compared with scrambled shRNA-

Table 1 Binding selectivity (geometric mean) of candidate podocalyxin antibodies compared with isotype control ${ }^{a}$

\begin{tabular}{llllllllll}
\hline mAb name & HUVEC & MCF7 & MDA-MB-231 & T47D & CAOV-3 & OVCAR-3 & OVCAR-10 & A-172 & HEK293 \\
\hline Isotype & 489 & 657 & 477 & 697 & 767 & 629 & 653 & 644 & 446 \\
PODOC1 & 44,611 & 60,805 & 91,909 & 1,498 & 48,817 & 16,502 & 2,834 & 169,600 & 28,225 \\
PODOC2 & 3,231 & 1,962 & 26,513 & 2,036 & 37,683 & 9,189 & 645 & 49,862 & 459 \\
PODOC3 & 2,103 & 9,399 & 2,939 & 818 & 10,472 & 8,355 & 1,803 & 181,211 & 3,869 \\
PODOC4 & 113,144 & 15,161 & 143,135 & 34,559 & 125,223 & 29,862 & 564 & 333,980 & 2,679 \\
PODOC5 & 40,490 & 25,055 & 80,312 & 1,853 & 31,657 & 9,824 & 1,756 & 172,233 & 27,144 \\
PODOC6 & 994 & 1,239 & 4,744 & 2,685 & 1,670 & 1,350 & 1,165 & 2,760 & 485 \\
PODOC7 & 21,700 & 22,330 & 57,626 & 1,010 & 37,668 & 11,058 & 2,539 & 278,032 & 13,886 \\
PODOC8 & 23,207 & 45,707 & 1,950 & 566 & 52,200 & 8,805 & 2,731 & 263,842 & 5,056 \\
\hline
\end{tabular}

aHEK293, Human embryonic kidney 293 cells; HUVEC, Human umbilical vascular endothelial cells; mAb, Monoclonal antibody; PODOC, Podocalyxin antibody. 


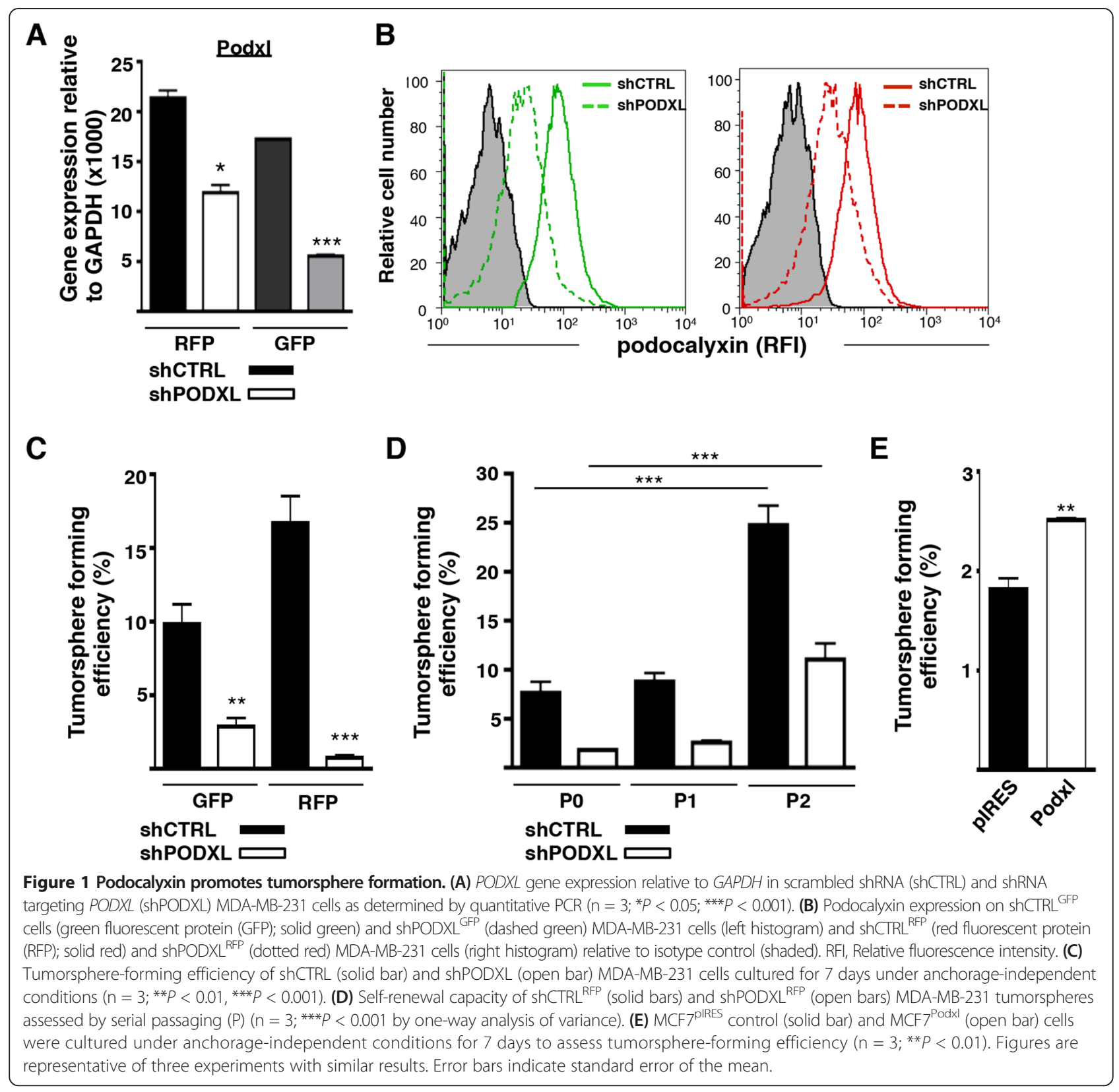

infected controls (shCTRL ${ }^{\text {GFP }}$; Figure 1B, left panel). Likewise, podocalyxin expression in shPODXL ${ }^{\mathrm{RFP}}$ cells was reduced by approximately twofold compared with shCTRL $^{\text {RFP }}$ control (Figure 1B, right panel). Similarly, total levels of podocalyxin protein were reduced in shPODXL cells compared with control as shown by Western blotting (Additional file 1).

Although proliferation of MDA-MB-231 cells in monolayer culture was unaffected by silencing $P O D X L$ (Additional file 2), the frequency of tumorsphereforming cells in three-dimensional assays was reduced by more than threefold in shPODXL cultures (Figure 1C). The comparable size (Additional file 3A, B) and morphology of control and shPODXL tumorspheres (Additional file $3 \mathrm{~B}$ ) suggests podocalyxin expression alters the frequency of tumorsphere-initiating cells rather than qualitatively affecting sphere formation per se. Serial passage of tumorsphere cultures is known to improve the efficiency of subsequent sphere formation [26,27], and both the shCTRL and shPODXL populations exhibited an approximately threefold increase in the frequency of sphere-initiating cells over three passages (Figure 1D). To further confirm that podocalyxin has a causal role in promoting tumorsphere formation in vitro, we overexpressed PODXL in MCF7 cells $\left(\mathrm{MCF}^{\text {Podxl}}{ }^{\text {) }}\right.$ (Additional file $3 \mathrm{C}$ ), a luminal-like human breast cancer cell line 
that expresses very low levels of endogenous podocalyxin $[10,19]$. MCF7 ${ }^{\text {Podxl }}$ cells (Additional file 3C) exhibited a 30\% increase in sphere-forming efficiency (Figure 1E). The results of these gain- and loss-offunction experiments suggest that podocalyxin expression increases the frequency of tumorsphere-forming cells in these cell lines. Because formation of tumorspheres in suspension culture provides an estimate of the frequency of tumor-initiating cells (TICs) [28-30], the observation that PODXL knockdown dampens tumorsphere formation is consistent with the notion that podocalyxin plays a role in TIC maintenance.

\section{Podocalyxin promotes primary tumor formation and metastasis}

To examine the functional significance of podocalyxin expression in vivo, immunocompromised mice (NSG strain) were given s.c. injections of shCTRL or shPODXL MDAMB-231 cells into the flank. Palpable solid tumors were detected 8 days after injection of shCTRL MDA-MB-231 cells, and these rapidly increased in volume over time. In comparison, growth of shPODXL MDA-MB-231 tumors was significantly attenuated. Tumors derived from shPODXL ${ }^{\text {RFP }}$ cells were less than $250 \mathrm{~mm}^{3}$ in volume by day 21, whereas shCTRL ${ }^{\text {RFP }}$ tumors reached sizes greater than $750 \mathrm{~mm}^{3}$ (Figure 2A, left). Similar results were obtained in independent experiments using shCTRL ${ }^{\mathrm{GFP}}$ and shPODXL ${ }^{\text {GFP }}$ MDA-MB-231 cells (Figure 2A, right). At the time the mice were killed, shCTRL tumors were 2.6-fold greater in mass than shPODXL tumors (Figure 2B). In addition, shPODXL tumors appeared to be more encapsulated, with little to no evidence of invasion into the surrounding skeletal muscle. In contrast, shCTRL tumors were highly invasive (Figure 2C, upper panel). Ki-67 expression was used to assess the level of proliferation of shCTRL and shPODXL tumor cells, and we observed a significant increase in cell proliferation (increased number of Ki-67-stained cells) in shCTRL tumors compared with shPODXL tumors (Figure 2C, lower panel), showing that $37.6 \pm 3.9 \%$ of shCTRL tumor cells were undergoing active proliferation, whereas no Ki-67-positive cells were detectable in shPODXL tumors. We also examined the lungs of these mice for signs of metastases 21 days after s.c. injection. Although no visible tumor nodules were observed, tumor cells were readily detectable by flow cytometry in single-cell suspensions from the lungs. Intriguingly, there was a 12-fold decrease in the frequency of metastatic cells in the lungs of mice with established shPODXL-derived primary tumors compared with mice with shCTRL-derived primary tumors (Figure 2D). We conclude that expression of podocalyxin enhances both primary tumor growth and metastasis in vivo.

\section{Podocalyxin enhances the metastatic potential of breast cancer cells}

To directly examine the functional significance of podocalyxin expression on lung-colonizing tumor cell frequency and metastatic behavior, we used an in vivo competitive assay (experimental lung metastasis). A 50:50 mixture of shPODXL and shCTRL MDA-MB-231 cells was injected into the tail vein of NSG mice, and relative tumor burden was evaluated at various time points over the next 6 weeks by tracking GFP or RFP expression (that is, tumor cells), respectively. Somewhat surprisingly, there was no significant difference in the ratio of ShPODXL to shCTRL cells recovered from the lung at days 3 and 7 postinjection. However, we observed a slight but significant reduction in the proportion of shPODXL cells colonizing the lung by day 14 (Figure 3A). Recovered tumor cells were evaluated for podocalyxin expression 3, 7 and 14 days after injection into the mice. Strikingly, all shPODXL cells began to reexpress podocalyxin within 7 days of injection (likely owing to the lack of drug selection required to maintain shRNA expression). By day 14, shPODXL cells expressed podocalyxin protein at levels similar to those of shCTRL tumors (Figure 3B). A similar result was obtained with primary solid tumors seeded s.c. in the flank (Additional file 4). Nevertheless, at 6 weeks post i.v. injection, there was a 2.3-fold reduction in shPODXL cell-derived tumor nodules on the lungs compared with competing shCTRL cell-derived tumors (Figure 3C). In addition, large, cancerous lesions were visible in the liver of some mice, and, invariably, all visible liver tumors were derived from shCTRL cells as determined by fluorescence microscopy (Figure 3D). Subsequent flow cytometric analyses of lung, liver and bone marrow single-cell suspensions also revealed a dramatic under-representation of shPODXL MDA-MB-231 cells colonizing these tissues (Figure 3E). Thus, although podocalyxin expression on MDA-MB231 cells does not alter initial "seeding" of the lung at early time points (up to 14 days), expression of podocalyxin greatly enhances subsequent establishment of clonal tumors in the lung and other organs. The similar size of the shCTRL- and shPODXL-derived lung nodules in these experimental assays (Additional file 5A) suggests that a difference in proliferation alone cannot account for the difference in the frequency of tumor nodules we observed. The higher frequency of shCTRL cells in the lungs correlates (Figure 3C) with the number of tumor nodules observed, rather than the size of the nodules.

To further corroborate these findings and evaluate the functional significance of podocalyxin on tumors in an immunocompetent host, we used a syngeneic model of tumor growth and metastasis. Using shRNA, we silenced mouse podocalyxin surface protein expression by 6.3- 


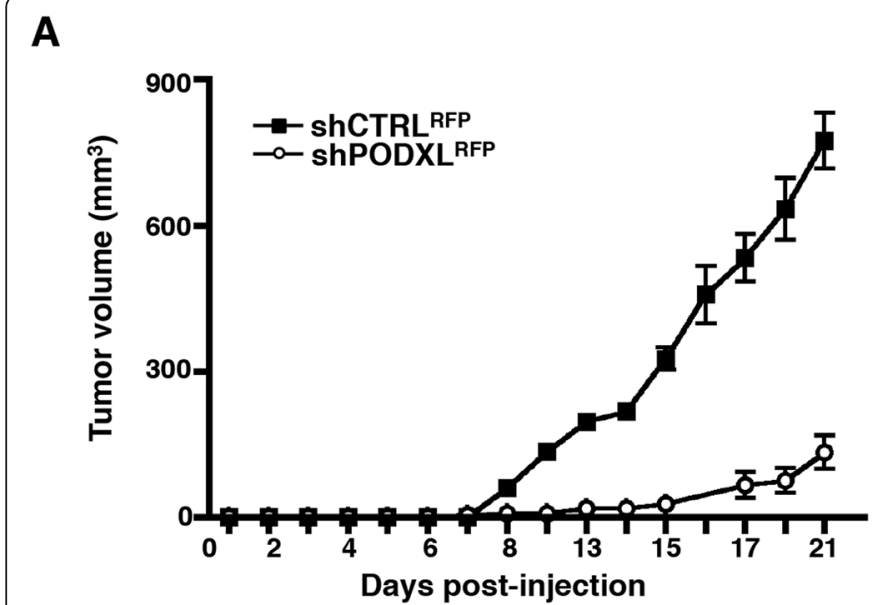

B

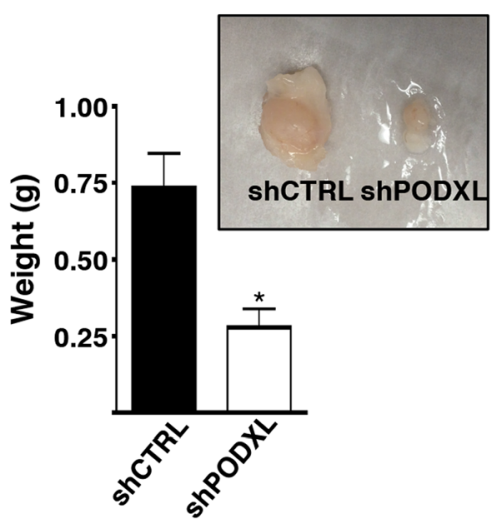

C

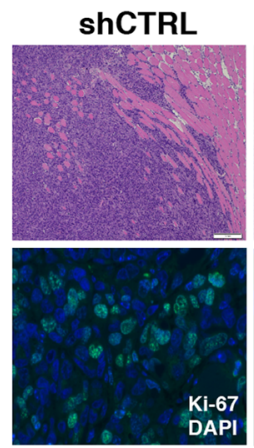

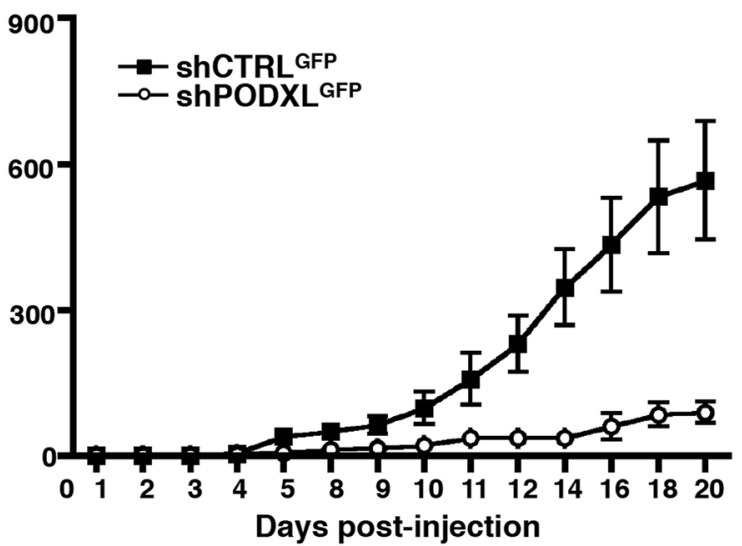

D
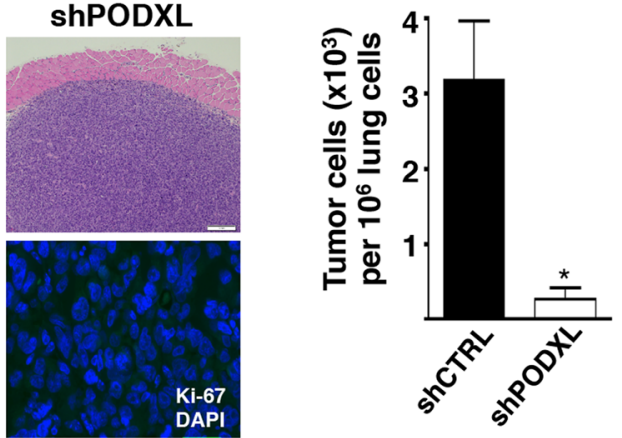

Figure 2 Podocalyxin promotes primary tumor formation, local invasion and metastasis. (A) Growth curve of subcutaneous (s.c.) flank tumors initiated in NSG mice by scrambled shRNA control (shCTRL ${ }^{\text {RFP }}$; closed squares) or shRNA targeting PODXL (shPODXL ${ }^{\text {RFP }}$; open circles) MDA-MB-231 cells (left; $\mathrm{n}=5$ for shCTRL ${ }^{\mathrm{RFP}}$ group and $\mathrm{n}=6$ for shPODXL ${ }^{\text {RFP }}$ group; growth curves are significantly different with $\left.P<0.001\right)$. Similarly, we compared s.c. tumor growth of shCTRL GFP with shPODXL ${ }^{\text {GFP }}$ MDA-MB-231 cells in NSG mice (right; $n=6$; growth curves are significantly different with $P<0.05$ ). GFP, Green fluorescent protein; RFP, Red fluorescent protein. The final volume of the excised tumors was measured on the dates mice were killed (left; day 21, right; day 20). (B) Wet weight (g) of pooled shCTRL (solid bar) and shPODXL (open bar) tumors weighed immediately after excision ( $\mathrm{n}=11$ for shCTRL group and $n=5$ for shPODXL group; ${ }^{*} P<0.05$ ). Representative photograph of excised shCTRL and shPODXL tumors (inset). (C) Representative H\&E-stained images of shCTRL (upper left) and shPODXL (upper right) primary tumor sections (scale bar $=1$ mm). Representative immunofluorescence images of shCTRL (lower left) and shPODXL (lower right) primary tumor sections showing Ki-67-positive cells (green). DAPI stain was used as a nuclear marker (blue). (D) The number of tumor cells detected in the lungs 20 or 21 days after subcutaneous injection. Data shown are the number of fluorescent tumor cells $\left(\times 10^{3}\right)$ per $10^{6}$ events detected by flow cytometry $\left(n=11\right.$ for shCTRL group and $n=5$ for shPODXL group; $\left.{ }^{*} P<0.05\right)$. The data shown are representative of three independent experiments.

fold in 4T1-luc mouse mammary tumor cells (BALB/c mouse-derived) (Figure 4A). Similar to our results with MDA-MB-231 cells, loss of podocalyxin expression in 4T1 cells also impaired the metastatic potential of these cells in an experimental model of lung metastasis using immunocompetent BALB/c mice (greater than threefold reduction), as shown by manual counts of tumor nodules on the lungs (Figure 4B) and by luciferase assays of lung homogenates (Figure 4C). As in the MDA-MB-231 experimental metastasis assay, the frequency of tumor nodules observed in $\mathrm{BALB} / \mathrm{c}$ recipients (rather than the size of the tumor nodules) (Figure $4 \mathrm{~B}$ and Additional file $5 \mathrm{~B}$ ) was affected by silencing Podxl expression in
4T1 cells. Again, the total tumor cell numbers in the lung (as measured by luciferase assay (Figure 4C)) was proportional to the frequency of tumor nodules. Thus, in both murine and human breast cancer cell lines, podocalyxin expression enhances experimental metastatic disease.

A novel podocalyxin-specific antibody prevents primary tumor growth in vivo

The finding that podocalyxin expression is capable of driving breast tumor progression encouraged us to evaluate the possibility that mAbs targeting the extracellular domain of podocalyxin would prove efficacious in delaying tumor 


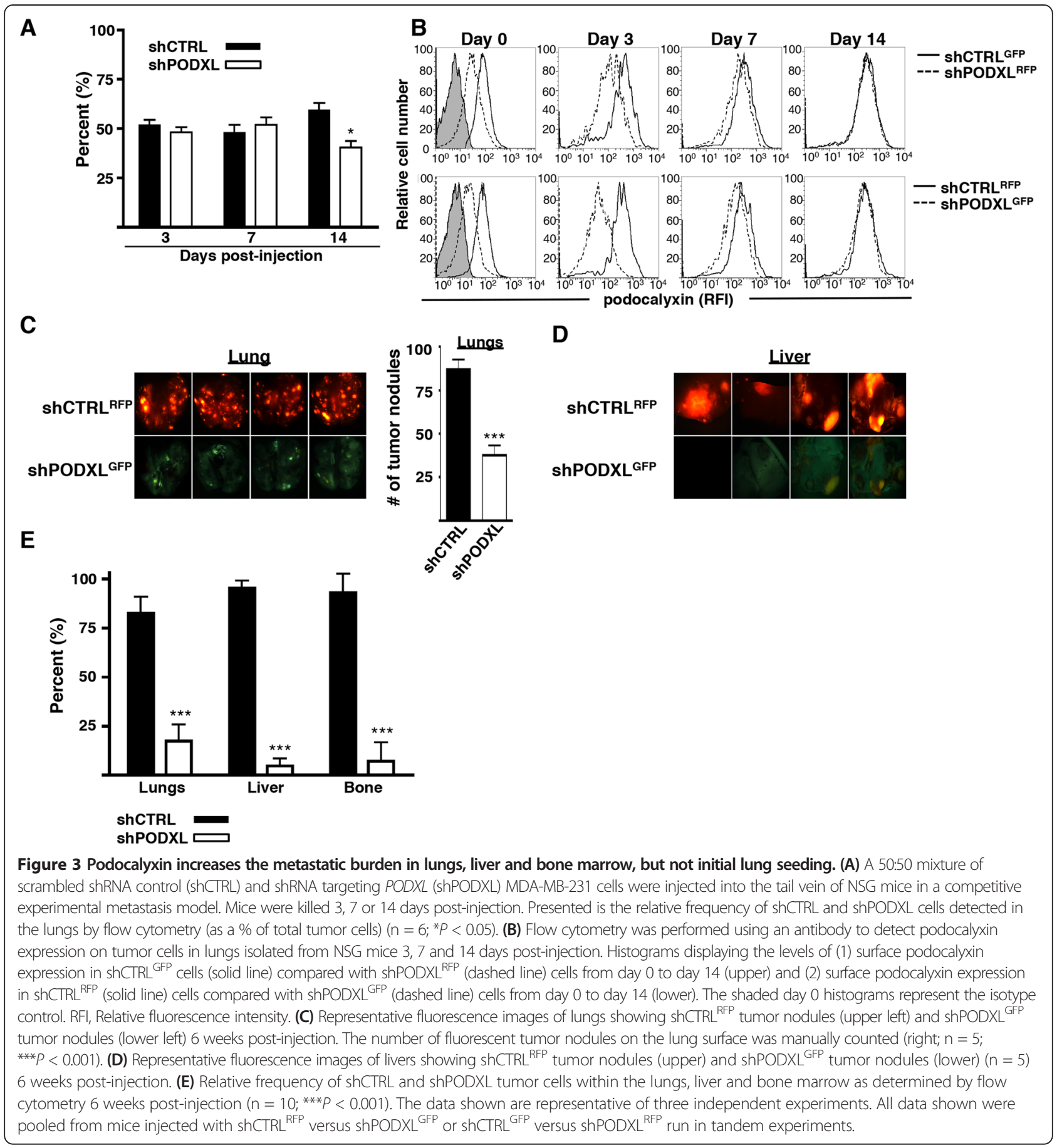

growth and metastasis. Using podocalyxin-expressing A172 glioblastoma cells as an immunogen, we generated a novel panel of anti-human podocalyxin mAbs that exhibit preferential binding to podocalyxin expressed on human tumor cells. Of these candidates, we selected eight mAbs (PODOC1 through PODOC8) with favorable selectivity profiles based on flow cytometry screening of tumor cell lines known to highly express podocalyxin (MDA-MB231, CAOV-3, A-172), tumor cell lines known to express low levels of podocalyxin (MCF7, T47D, OVCAR-10) and non-tumor-derived human cells known to express podocalyxin (human umbilical vascular endothelial cells and HEK293 cells) (Table 1). Although we generated several antipodocalyxin mAbs with affinity for podocalyxin 

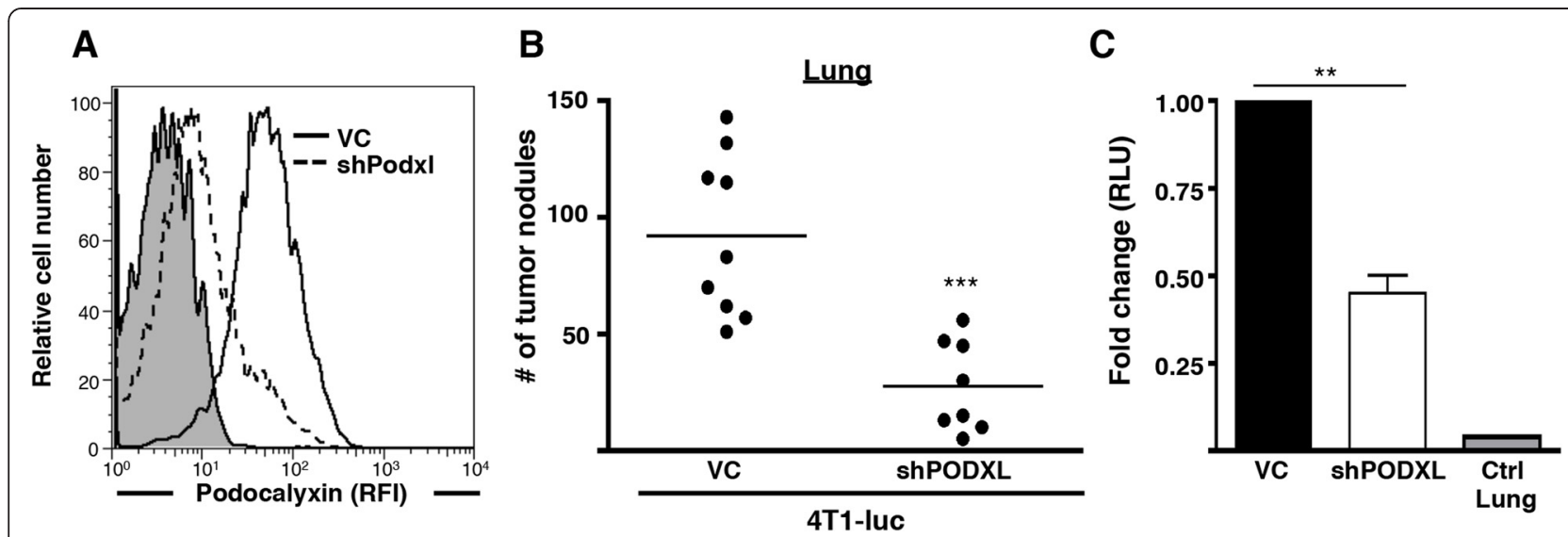

Figure 4 Podocalyxin enhances metastasis of 4T1 mammary tumor cells in a syngeneic mouse model. (A) Vector control (VC, black) and shRNA targeting PODXL (shPODXL; dashed line) luciferase-expressing 4T1 (4T1-luc) murine mammary tumor cells were labeled with a conjugated rat anti-mouse podocalyxin antibody and analyzed by flow cytometry to detect surface expression of podocalyxin. Rat ig $\mathrm{G}_{2 B}$ was used as an isotype control (shaded area). Data presented as histograms showing podocalyxin expression on VC cells compared with shPODXL 4T1-luc cells. RFI, Relative fluorescence intensity. (B) VC or shPODXL 4T1-luc cells were injected intravenously into BALB/c mice. Mice were killed after 14 days, and visible tumor nodules on the lung were manually counted using a dissecting microscope $(n=9$ and $n=8$, respectively; ***P < 0.001). (C) Luciferase expression in the lungs from noninjected, VC and shPODXL 4T1-luc-injected mice was measured using a luminometer $\left({ }^{* *} P<0.01\right)$. The data shown are representative of two independent experiments. RLU, Relative light units.

expressed on MDA-MB-231 cells, none of these exhibited an effect on tumor cell growth in monolayers or tumorsphere formation in vitro (data not shown).

Nevertheless, because podocalyxin expression appears to predominantly affect the ability of tumor cells to colonize tissues in vivo (rather than influencing their behavior in vitro), we went on to evaluate the effects of these antibodies in xenograft assays. Candidate mAbs were incubated with tumor cells immediately before s.c. injection into the flanks of recipient mice (pretreatment screen). One antipodocalyxin mAb (PODOC1) inhibited MDA-MB231 growth and dissemination for 11 days (Figure 5A), whereas the Iso and 7 other podocalyxin-binding candidate mAbs (PODOC2 through PODOC8) failed to significantly alter tumor progression (Figure 5, B-H). Next, we assessed the ability of PODOC1 to inhibit established tumor growth (Figure 6). Cohorts of mice were treated therapeutically with PODOC1 or control Ab beginning 14 days after tumor injection, when a palpable tumor had already formed (s.c.). We consistently found that systemic treatment of tumor-bearing mice with PODOC1 completely inhibited tumor growth (Figure 6A, B) and, importantly, attenuated micrometastases to the lung (Figure 6C). Thus, we validated podocalyxin as a critical facilitator of tumor growth and progression and a therapeutic target for treatment in a preclinical model. To confirm that PODOC1 specifically binds to podocalyxin, Western blot analysis was performed on shCTRL and shPODXL MDA-MB-231 cell lysates. PODOC1 detected high levels of podocalyxin protein in shCTRL cells and significantly less in shPODXL cells (Additional file 6A). Additionally, in flow cytometric analyses, PODOC1 detected extracellular podocalyxin on shCTRL and lower levels on shPODXL MDA-MB-231 cells (Additional file 6B). It was also important to determine whether PODOC1 was specific for podocalyxin and did not bind to the closely related family member, CD34. Utilizing $\mathrm{CHO}$ cells transfected with human podocalyxin or CD34 (hCD34), PODOC1 was found to specifically detect overexpression of podocalyxin (Additional file 7B), whereas it failed to bind to hCD34 (Additional file 7C) or mock-transfected $\mathrm{CHO}$ cells (Additional file 7A). Thus, our data would support the argument that the PODOC1 $\mathrm{Ab}$ is highly specific and does not cross-react with a closely related sialomucin. We conclude that antibodies targeting the appropriate epitope on human podocalyxin can provide therapeutic benefit in vivo.

Finally, to test the potential efficacy of PODOC1 on late-stage metastatic disease, mice were injected s.c. with shCTRL ${ }^{\text {GFP }}$ MDA-MB-231 cells and tumors were allowed to reach a size larger than $500 \mathrm{~mm}^{3}$ prior to PODOC1 therapy. At this size, we find that metastatic lesions readily develop in the lungs. PODOC1 or Iso $\mathrm{Ab}$ was then administered to the mice with established tumor burdens at days 20, 26, 29 and 32 (Additional file 8A). Systemic treatment with PODOC1 appeared to marginally slow the growth of the primary tumor, although the difference was not statistically significant (Additional file $8 \mathrm{~A}, \mathrm{~B})$. However, PODOC1 treatment resulted in a dramatic reduction in the number of tumor nodules observed on the lung surface (Additional file $8 \mathrm{C}$ ) recovered in total lung homogenates (Additional file 8D). We conclude that PODOC1 provides a clear therapeutic benefit, even in late-stage metastatic disease (that is, 

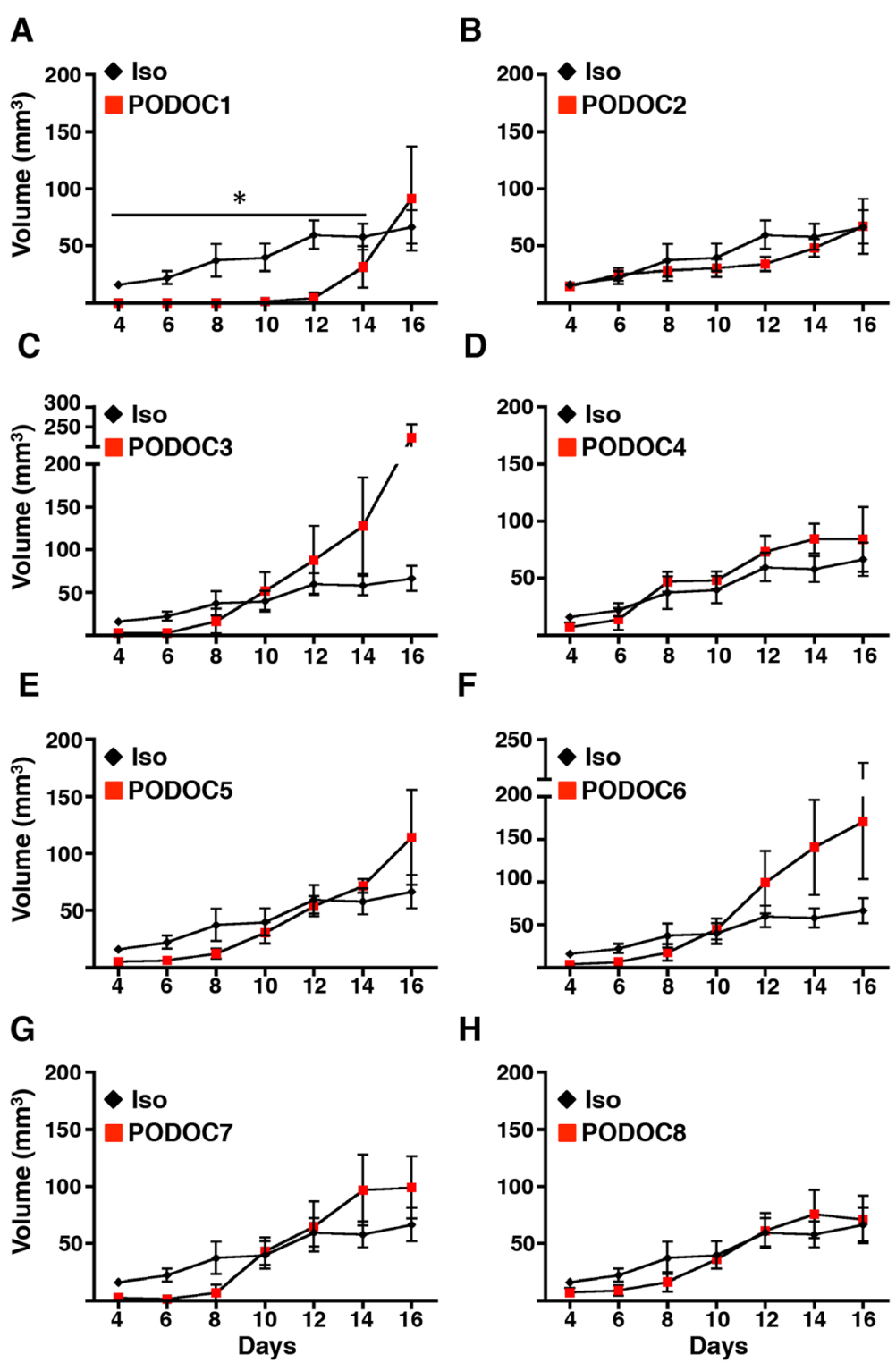

H

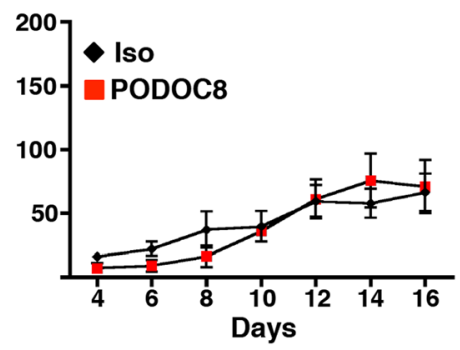

Figure 5 Pretreatment of MDA-MB-231 cells with PODOC1 delays primary tumor development. Growth curves from days 4 to 16 (post-injection) of tumors resulting from subcutaneously injected MDA-MB-231 cells pretreated with isotype control (Iso; red) or one of eight novel candidate antipodocalyxin antibodies (PODOC). (A) PODOC1 Ab ( $\left.{ }^{*} \mathrm{p}<0.05 ; \mathrm{n}=3\right)$. (B) through $(\mathbf{H})$ Growth curves for other candidate PODOCs (PODOC2 through PODOC8; $n=3$ ). The data shown are representative of two independent experiments.

when metastatic organs are already colonized with tumor cells).

\section{Discussion}

Although podocalyxin is expressed by a minor subset of primary breast tumors, these have been shown to be the most aggressive and difficult-to-treat breast cancers [10]. Importantly, podocalyxin expression is also a predictor of poor prognosis in many other cancers $[10-15,17,18,20,31,32]$. For example, patients with podocalyxin-positive colorectal carcinoma (where podocalyxin-expressing cells are often located at the invasive front of the primary tumor) have a higher probability of lymph node and distant metastases [31]. In addition, roughly $20 \%$ of stage III colorectal carcinomas express high levels of podocalyxin, and these represent a cohort that significantly benefits from adjuvant chemotherapy [31]. Comparatively, similar patients with low levels of tumor podocalyxin did not appear to significantly benefit from chemotherapy [31]. Knowing the likelihood of success before accepting a treatment that is difficult for some patients to tolerate has obvious decision-making benefit. Thus, podocalyxin-based "theranostic" and therapeutic strategies may prove to have broad applications if podocalyxin promotes primary tumor growth and metastasis in colorectal 

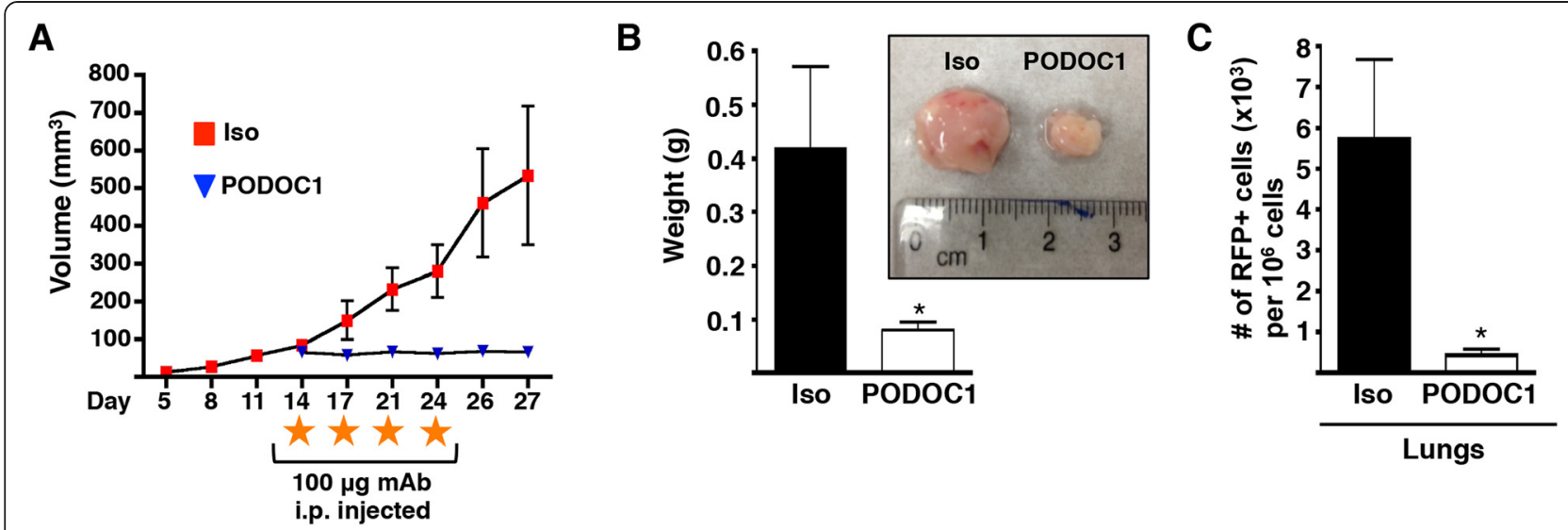

Figure 6 Systemic treatment with antipodocalyxin antibody PODOC1 inhibits primary tumor development and metastasis to the lung. (A) Growth curve of tumors from mice treated intraperitoneally (i.p.) with $100 \mu \mathrm{g}$ of isotype control (Iso) or antipodocalyxin monoclonal antibody (mAb) PODOC1 on day 14 and every 3 to 4 thereafter days until being killed on day 27 ( $n=5$; ${ }^{*} P<0.05$ by two-way analysis of variance) (right). Orange stars indicate i.p. administration of antibody. (B) Weight ( $\mathrm{g}$ ) of tumors treated with either Iso or PODOC1. Representative photograph shows tumors treated with either Iso or PODOC1 (inset) ( $\left.\mathrm{n}=5 ;{ }^{*} \mathrm{P}=0.05\right)$. (C) Number of red fluorescent protein (RFP)-positive tumor cells per $10^{6}$ lung cells of mice with tumors treated i.p. with either Iso or PODOC1 as detected by flow cytometry $\left(n=5\right.$; $\left.{ }^{*}<0.05\right)$. The data shown are representative of two independent experiments.

carcinoma and other epithelial cancers. It is now important to further explore the therapeutic efficacy of PODOC1 and similar reagents in a clinical setting. Because podocalyxin is present on normal human cells, including the vascular endothelium and kidney podocytes, extensive toxicologic studies will be needed to ensure the safety of a therapeutic Ab. However, we predict that because the podocalyxin-rich podocytes of the kidney are behind the blood filtration barrier in the urinary space, they may be spared exposure to PODOC1 therapy. Additionally, we have not observed any adverse effect of antibodies targeting mouse podocalyxin when systemically administered to wild-type mice. Furthermore, we have found that selective deletion of Podxl from mouse endothelia is well tolerated and nontoxic in mice [33]. Thus, the data would support the argument that interfering with podocalyxin expression on endothelia or binding of podocalyxin-reactive antibodies to the vasculature is unlikely to be toxic.

By identifying a requirement for podocalyxin in tumorigenesis, we can now begin to characterize the key molecular mechanisms by which podocalyxin promotes tumor cell growth and colonization of supportive niches. As is highlighted by Ki-67 staining of subcutaneous tumors, one function of podocalyxin may be the promotion of primary tumor cell proliferation in vivo. Intriguingly, this effect was observed only in vivo because loss of podocalyxin had no effect on the proliferation of cultured tumor cells. Notably, although silencing podocalyxin is detrimental to tumorsphere-forming efficiency of MDA-MB-231 cells, the PODOC1 mAb does not appear to alter tumorsphereforming efficiency or proliferation in vitro (not shown). Tumor formation and metastasis in vivo are dependent on a number of cellular characteristics that are difficult to mimic in vitro, including migration to and colonization of a supportive niches, immune cell evasion, and survival of a hypoxic environment until the establishment of an adequate blood supply. It is likely that podocalyxin functions in these settings are multifactorial, and, thus far, we have been compelled to use in vivo models for these studies. It is now important to evaluate the molecular pathways podocalyxin impinges on in vivo that lead to altered tumor cell proliferation.

With regard to the early stages of tumor colonization of tissues, it is intriguing that the bulk of our shPODXL cells begin to reexpress PODXL during the first 7 to 14 days in vivo. Thus, our data would support an argument for an important influence of podocalyxin on an early tumor-initiating subset of cells. This notion is supported by the fact that, in our experimental lung metastasis assays, we found that silencing podocalyxin expression decreased the frequency (but not the size) of tumor nodules we observed. In aggregate, these data suggest that even transient depletion of podocalyxin expression during the early phase of tumor establishment can have a profound effect on late-stage growth of metastases, perhaps through impaired function or decreased frequency of a population of cells with TIC-like properties. We do not yet know which properties of TICs are influenced by podocalyxin expression, but they could include properties that enable tumor cells to proliferate or survive within a metastatic niche, including invasion, migration, adhesion and recruitment of supportive vasculature. It is noteworthy that podocalyxin, and its close relative CD34, are well-known markers of various subsets of stem cells during development and play a role in cell and tissue morphogenesis and colonization of developing tissues $[7,9,26,27,31]$. Likewise, podocalyxin was recently detected in an undifferentiated stemlike 
population in glioblastoma multiforme [11], and it is a well-known marker of both embryonic stem cells and embryocarcinomas $[3,6]$. Thus, in both normal development and neoplastic disease, podocalyxin expression has been linked to stem cell activity. Impaired tumor initiation would be consistent with known roles for podocalyxin and CD34-type proteins in blocking cell adhesion and facilitating chemokine-dependent inflammatory trafficking and hematopoietic stem cell engraftment of the bone marrow niche [34-36]. Importantly, in contrast to the wide variety of drugs that target tumor proliferation, there is a paucity of therapeutics that target TIC activity, and therefore the Ab strategy described here may be an important additional therapeutic avenue.

In many ways, our findings are complementary to those described in a recent publication by Lin et al. [37]. These authors provided provocative evidence that both podocalyxin and cortactin are important for the morphogenesis, motility, gelatin invasion and in vivo metastatic potential of MDA-MB-231 cells and showed that these proteins associate in vitro. Although they did not show that podocalyxin is essential for cortactin-mediated metastasis in vivo, these data do offer a potential mechanistic insight into podocalyxin function through a cortactincontaining complex. Given that our present study shows podocalyxin to be functionally important for tumorsphereforming cells in vitro and the early phases of tumor colonization by a subset of cells in vivo, it is now important to validate the functional significance of the cortactin and podocalyxin interaction in this rare, but clinically critical, subset of tumor cells.

\section{Conclusions}

It has previously been shown that podocalyxin expression in invasive breast carcinoma correlates with poor patient survival and that podocalyxin enhances the motility and invasiveness of breast cancer cell lines in vitro [10,19-21]. Here, using in vivo models of breast tumor growth and metastasis, we show that podocalyxin has a causal role in promoting the growth and proliferation of solid tumors and enhancing the metastasis of tumor cells to distant organs. We found that silencing podocalyxin expression in MDA-MB-231 cells, an aggressive triple-negative, basal-like breast cancer cell line, severely impaired primary tumor growth and metastasis to the lung, liver and bone marrow in a xenograft model. We corroborated these results in a syngeneic mouse model using fully immunocompetent mice by silencing podocalyxin expression in mouse mammary tumor $4 \mathrm{~T} 1$ cells. Thus, in both mouse and human breast tumor cells, podocalyxin plays a critical role in disease progression. Furthermore, we have developed a unique $\mathrm{mAb}$ that targets podocalyxin and, in preclinical mouse studies, inhibits tumor growth and metastatic progression.

\section{Additional files}

Additional file 1: Podocalyxin expression can be efficiently knocked down in MDA-MB-231 cells. shCTRL and shPODXL MDA-MB-231 whole-cell lysates $\left(5 \times 10^{4}\right.$ cells) were resolved by SDS-PAGE and analyzed by Western blotting using antipodocalyxin clone 3D3 (1:4,000; Santa Cruz Biotechnologies, Santa Cruz, CA, USA). An antibody against $\beta$-actin (1:10,000; Sigma-Aldrich) was used as a loading control.

Additional file 2: Proliferation is not affected by podocalyxin expression in MDA-MB-231 cells in monolayer culture. A 3-(4,5dimethylthiazol-2-yl)-5-(3-carboxymethoxyphenyl)-2-(4-sulfophenyl)-2 $\mathrm{H}$ tetrazolium proliferation assay was performed on shCTRL and ShPODXL MDA-MB-231 cells 48, 72 and 96 hours after initial seeding. Proliferation was quantified by the amount of formazan product detected at 490-nm absorbance using a microplate reader. The level of proliferation of ShCTRL was compared with ShPODXL cells over time (nonsignificant by two-way analysis of variance). All values are graphed as mean \pm SEM.

Additional file 3: shCTRL and shPODXL cells form tumorspheres of similar size and morphology. A total of $5 \times 10^{3}$ shCTRL or ShPODXL MDA-MB-231 cells were cultured for 7 days in MammoCult medium. (A) Tumorsphere size was calculated using ImageJ software (pixels). (B) Representative images of shCTRL and shPODXL MDA-MB-231 tumorspheres. (C) Murine podocalyxin expression can be overexpressed in human MCF7 breast tumor cells. MCF7 ${ }^{\text {IRES }}$ control and MCF7 Podxl lysates were resolved by SDS-PAGE and analyzed by Western blotting using a goat anti-mouse podocalyxin antibody ( $1 \mu \mathrm{g} / \mathrm{ml}$; R\&D Systems). An antibody against $\beta$-actin was used as a loading control. (D) Flow cytometry was performed to detect the level of extracellular murine podocalyxin on MCF7 ${ }^{\text {plRES }}$ (left; blue) and MCF7 ${ }^{\text {Podxl }}$ cells (right; red). A goat anti-mouse podocalyxin antibody $\left(2.5 \mu \mathrm{g} / 10^{6}\right.$ cells; R\&D Systems) and an isotype against normal goat lgG (gray) were used.

Additional file 4: Podocalyxin is reexpressed in primary MDA-MB231 tumors after 14 days in vivo. A total of $1 \times 10^{6}$ shCTRL $^{\text {GFP }}$ or shPODXL GFP MDA-MB-231 cells were injected s.c. into the right and left flanks of NSG mice. After 14 days, mice were killed and perfused with 10 $\mathrm{ml}$ of ice-cold PBS, and tumors were excised. (A) One-third of each ShCTRL and ShPODXL tumor was processed for flow cytometric analysis using a $2-\mathrm{U} / \mathrm{ml}$ collagenase solution for 1 hour at $37^{\circ} \mathrm{C}$ and stained for extracellular podocalyxin using one of two antibodies (PODOC1 or goat antihuman podocalyxin; R\&D Systems). The upper two histograms display the level of surface podocalyxin expression in shCTRL GFP cells (left; blue) compared with shPODXL GFP cells (right, blue) as detected by PODOC1 antibody. A goat antihuman AF647 ( $2 \mu \mathrm{g} / \mathrm{ml}$; Invitrogen) secondary-only control is shown in red. The lower two histograms display the level of surface podocalyxin expression in shCTRL GFP cells (left; blue) compared with shPODXL GFP cells (right; blue) as detected by goat antihuman podocalyxin ( $2 \mu \mathrm{g} / \mathrm{ml}$; R\&D Systems) followed by chicken anti-goat AF647 (Invitrogen). Normal goat lgG isotype control is shown in red. (B) One-third of the tumors were processed "fresh" for Western blot analysis by directly homogenizing them in $500 \mu$ l of radioimmunoprecipitation assay (RIPA) lysis buffer, and the final third of the tumors were freeze-thawed $(F / T)$ by snap-freezing in dry ice and storing them at $-80^{\circ} \mathrm{C}$ for 1 hour. $F / T$ tumors were homogenized in $500 \mu \mathrm{l}$ of RIPA lysis buffer. A BCA assay was performed, and equal amounts of protein were resolved by SDS-PAGE and analyzed by Western blotting using antipodocalyxin clone 3D3 (1:4,000; Santa Cruz Biotechnologies). An antibody against $\beta$-actin (1:10,000; Sigma-Aldrich) was used as a loading control.

Additional file 5: Metastatic lung nodules resulting from MDA-MB231 cells or 4D1 cells are more prevalent in number, but not of greater size when compared with their respective podocalyxin knockdown lines. (A) NSG mice were injected (i.v.) with a 50:50 mixture of shCTRL GFP and ShPODXL RFP MDA-MB-231 cells $\left(5 \times 10^{4}\right.$ cells). After 6 weeks, mice were killed and their lungs and fluorescent nodules on the lungs were imaged using a fluorescence dissecting microscope. The GFP and RFP channels were merged as a composite image to show nodules arising from both shCTRL and shPODXL cells. (B) BALB/c mice were injected (i.v.) with $1 \times 10^{5}$ vector control (VC) or shPodxl mouse murine 4D1 tumor cells. After 2 weeks, lungs were perfused with ice-cold PBS, fixed in $10 \%$ buffered formalin, embedded in paraffin and sectioned. 
Representative lung sections containing VC or shPodxl nodules were stained with $\mathrm{H} \& \mathrm{E}$.

Additional file 6: PODOC1 antibody specifically detects podocalyxin expression in MDA-MB-231 cells. (A) ShCTRL and ShPODXL MDA-MB-231 whole-cell lysates were resolved by SDS-PAGE and analyzed by Western blotting using candidate therapeutic antibody PODOC1 $(1 \mathrm{\mu g} / \mathrm{ml})$. An antibody against $\beta$-actin was used as a loading control. (B) Podocalyxin expression on shCTRL ${ }^{\text {GFP }}$ (solid) and shPODXL ${ }^{\text {GFP }}$ (dashed) MDA-MB-231 cells relative to secondary-only control (shaded), as detected using PODOC1 $(10 \mu \mathrm{g} / \mathrm{ml})$ antibody followed by goat antihuman AF647 secondary antibody ( $2 \mu \mathrm{g} / \mathrm{ml}$; Invitrogen) (right).

\section{Additional file 7: PODOC1 antibody specifically interacts with} podocalyxin and does not bind to CD34. Human CD34 (hCD34) and podocalyxin were transiently overexpressed in $\mathrm{CHO}$ cells. Flow cytometry was performed on $\mathrm{CHO}$ cells transfected with (A) human podocalyxin $\left(\mathrm{CHO}{ }^{\mathrm{PODXL}}\right)$ or (B) human CD34 (CHO $\left.{ }^{\text {hCD34 }}\right)$ or (C) mock sequence $\left(\mathrm{CHO}^{\text {Mock }}\right)$ and stained with either PODOC1 (left) $(10 \mu \mathrm{g} / \mathrm{ml})$ or mouse antihuman CD34 fluorescein isothiocyanate-conjugated antibody (right) (1:50; Invitrogen).

Additional file 8: Systemic treatment with PODOC1 inhibits metastasis to the lung in mice with large $\left(>500 \mathrm{~mm}^{3}\right)$ primary MDA-MB-231 tumors. A total of $1 \times 10^{6}$ shCTRL ${ }^{\text {GFP }}$ MDA-MB-231 cells were injected s.c. into the flank of NSG mice and allowed to develop into solid tumors over 20 days. (A) Growth curve of tumors from mice treated intraperitoneally (i.p.) with $4.5 \mathrm{mg} / \mathrm{kg}$ of isotype control (Iso) or PODOC1 $\mathrm{Ab}$ on day 20 and at three time points until the mice were killed on day 36 (nonsignificant (n.s.) by two-way analysis of variance; $n=5$ ) (right). Orange stars indicate i.p. administration of antibody. (B) Weight (g) of tumors treated with either Iso or PODOC1 antibody ( $n=5 ; n . s$. by Student's $t$-test). (C) Representative bright-field and fluorescence microscopic images of lungs showing shCTRL GFP tumor nodules from mice that had been systemically treated with Iso (upper two panels) or PODOC1 (lower two panels). (D) Percentage of GFP-positive tumor cells in the lungs of mice with tumors treated i.p. with either isotype or PODOC1 antibody as detected by flow cytometry $\left(n=5\right.$; $\left.{ }^{*} P<0.05\right)$.

\section{Abbreviations}

Ab: Antibody; AF: Alexa Fluor; CHO: Chinese hamster ovary cells; DMEM: Dulbecco's Modified Eagle's medium;

EDTA: Ethylenediaminetetraacetic acid; ER: Estrogen receptor; FBS: Fetal bovine serum; F/T: Freeze-thawed; GFP: Green fluorescent protein; H\&E: Hematoxylin and eosin; HEK293: Human embryonic kidney 293; HER2: Human epidermal growth factor receptor 2; HUVEC: Human umbilical vascular endothelial cell; IgG: Immunoglobulin G; i.p.: Intraperitoneal; Iso: Isotype; i.v: Intravenous; mAb: Monoclonal antibody; MEP21: Myb-Ets progenitor 21; NSG: Nonobese diabetic severe combined immunodeficiency, interleukin 2 gamma chain deficiency, NOD.Cg-Prkd $c^{\left.\text {cid } / / 2 r g^{t m} 1 W_{j i l} / S z\right] ; ~}$ PBS: Phosphate-buffered saline; PCLP1: Podocalyxin-like protein 1; PgR: Progesterone receptor; PODO: Podocalyxin; RFI: Relative fluorescence intensity; RFP: Red fluorescent protein; RIPA: Radioimmunoprecipitation assay; RLU: Relative light units; s.c.: Subcutaneous; SEM: Standard error of the mean; shCTRL: Scrambled short-hairpin RNA control; shPODXL: Short-hairpin RNA targeting PODXL; shRNA: Short-hairpin RNA; 4T1-luc: Luciferase-expressing 4T1 cells; TIC: Tumor-initiating cell; VC: Vector control.

\section{Competing interests}

The authors (KAS, MRH, KMM, CDR, JSB, BH and MLG) declare that they have a patent pending on podocalyxin as potential therapeutic for epithelial tumors. KMM and CDR possess an awarded US patent on podocalyxin as a prognostic marker in cancer.

\section{Authors' contributions}

KS and MRH carried out all experiments, participated in the design and coordination of the study and drafted the manuscript. BH, JB, PB, FC, KP and JSB developed and produced candidate antipodocalyxin antibodies and contributed intellectually to the conception and design of the antibody studies. MET and SRM knocked down PODXL in MDA-MB-231 cells using vector provided by JAW and contributed intellectually to all of the MDA-MB-231 studies. JAW contributed to the experimental design of the MDA-MB-231 studies. DCH carried out xenograft assays together with $\mathrm{KS}$ and contributed intellectually to antipodocalyxin antibody studies. MLG and JSN developed the podocalyxin overexpressing the MCF7 cell line and the murine 4T1 knockdown cell line and contributed intellectually to the design of these studies. KMM and CDR conceived of the study, participated in its design and coordination and helped to draft the manuscript. All authors read and approved the final manuscript.

\section{Acknowledgments}

We are very grateful to Drs Martin Lopez, Megan Gilmour and Pamela Dean for providing expert technical assistance and generating the Western blot data. Thank you to Dr Matthew Gold for his critical reading of the manuscript. DCH received a graduate student fellowship from the Centre for Blood Research, University of British Columbia. This work was supported by an operating grant from the Canadian Institutes of Health Research (KMM, MOP\# 125992), an Impact Grant from the Stem Cell Network Centre of Excellence (KMM), and funding from National Health and Medical Research Council (SRM)

\section{Author details}

${ }^{1}$ The Biomedical Research Centre, University of British Columbia, 2222 Health Sciences Mall, Vancouver, BC V6T 1Z3, Canada. ${ }^{2}$ Centre for Drug Research and Development, University of British Columbia, Vancouver, BC V6T 1Z3, Canada. ${ }^{3}$ Department of Cellular and Physiological Sciences, University of British Columbia, Vancouver, BC V6T 1Z3, Canada. ${ }^{4}$ Centre for Molecular Pathology, School of Molecular \& Biological Science, The University of Adelaide, Adelaide, SA 5005, Australia. ${ }^{5}$ Department of Internal Medicine, Manitoba Centre for Proteomics and Systems Biology, University of Manitoba, Winnipeg, MB, Canada.

Received: 22 June 2014 Accepted: 17 March 2015

Published online: 27 March 2015

\section{References}

1. Weigelt $B$, Peterse $J$, van 't Veer $L J$. Breast cancer metastasis: markers and models. Nat Rev Cancer. 2005;5:591-602.

2. Dent R, Trudeau M, Pritchard KI, Hanna WM, Kahn HK, Sawka CA, et al. Triple-negative breast cancer: clinical features and patterns of recurrence. Clin Cancer Res. 2007;13:4429-34.

3. Schopperle WM, DeWolf WC. The TRA-1-60 and TRA-1-81 human pluripotent stem cell markers are expressed on podocalyxin in embryonal carcinoma. Stem Cells. 2007;25:723-30.

4. Kelley TW, Huntsman D, McNagny KM, Roskelley CD, Hsi ED. Podocalyxin: a marker of blasts in acute leukemia. Am J Clin Pathol. 2005;124:134-42.

5. Graf T, McNagny K, Brady G, Frampton J. Chicken "erythroid" cells transformed by the Gag-Myb-Ets-encoding E26 leukemia virus are multipotent. Cell. 1992;70:201-13.

6. Schopperle WM, Kershaw DB, DeWolf WC. Human embryonal carcinoma tumor antigen, Gp200/GCTM-2, is podocalyxin. Biochem Biophys Res Commun. 2003;300:285-90

7. Doyonnas R, Kershaw DB, Duhme C, Merkens H, Chelliah S, Graf T, et al. Anuria, omphalocele, and perinatal lethality in mice lacking the CD34-related protein podocalyxin. J Exp Med. 2001;194:13-27.

8. Nielsen JS, MCNagny KM. The role of podocalyxin in health and disease. J Am Soc Nephrol. 2009;20:1669-76.

9. Strilic B, Kucera T, Eglinger J, Hughes MR, McNagny KM, Tsukita S, et al. The molecular basis of vascular lumen formation in the developing mouse aorta. Dev Cell. 2009:17:505-15.

10. Somasiri A, Nielsen JS, Makretsov N, McCoy ML, Prentice L, Gilks CB, et al. Overexpression of the anti-adhesin podocalyxin is an independent predictor of breast cancer progression. Cancer Res. 2004;64:5068-73.

11. Binder ZA, Siu IM, Eberhart CG, ap Rhys C, Bai RY, Staedtke V, et al. Podocalyxin-like protein is expressed in glioblastoma multiforme stem-like cells and is associated with poor outcome. PLoS One. 2013;8:e75945.

12. Boman $\mathrm{K}$, Larsson $\mathrm{AH}$, Segersten $U$, Kuteeva $E$, Johannesson $H$, Nodin $B$, et al. Membranous expression of podocalyxin-like protein is an independent factor of poor prognosis in urothelial bladder cancer. Br J Cancer. 2013;108:2321-8.

13. Cipollone JA, Graves ML, Kobel M, Kalloger SE, Poon T, Gilks CB, et al. The anti-adhesive mucin podocalyxin may help initiate the transperitoneal metastasis of high grade serous ovarian carcinoma. Clin Exp Metastasis. 2012;29:239-52. 
14. Dallas MR, Chen SH, Streppel MM, Sharma S, Maitra A, Konstantopoulos K. Sialofucosylated podocalyxin is a functional E- and L-selectin ligand expressed by metastatic pancreatic cancer cells. Am J Physiol Cell Physiol. 2012;303:C616-24.

15. Hsu YH, Lin WL, Hou YT, Pu YS, Shun CT, Chen CL, et al. Podocalyxin EBP50 ezrin molecular complex enhances the metastatic potential of renal cell carcinoma through recruiting Rac1 guanine nucleotide exchange factor ARHGEF7. Am J Pathol. 2010;176:3050-61.

16. Larsson A, Fridberg M, Gaber A, Nodin B, Leveen P, Jonsson G, et al. Validation of podocalyxin-like protein as a biomarker of poor prognosis in colorectal cancer. BMC Cancer. 2012;12:282.

17. Wu H, Yang L, Liao D, Chen Y, Wang W, Fang J. Podocalyxin regulates astrocytoma cell invasion and survival against temozolomide. Exp Ther Med. 2013:5:1025-9.

18. Yasuoka H, Tsujimoto M, Inagaki M, Kodama R, Tsuji H, Iwahashi Y, et al. Clinicopathological significance of podocalyxin and phosphorylated ezrin in uterine endometrioid adenocarcinoma. J Clin Pathol. 2012;65:399-402.

19. Nielsen JS, Graves ML, Chelliah S, Vogl AW, Roskelley CD, McNagny KM. The CD34-related molecule podocalyxin is a potent inducer of microvillus formation. PLoS One. 2007;2:e237.

20. Sizemore S, Cicek M, Sizemore N, Ng KP, Casey G. Podocalyxin increases the aggressive phenotype of breast and prostate cancer cells in vitro through its interaction with ezrin. Cancer Res. 2007;67:6183-91.

21. Meng X, Ezzati P, Wilkins JA. Requirement of podocalyxin in TGF- $\beta$ induced epithelial mesenchymal transition. PLoS One. 2011;6:e18715.

22. McNagny KM, Hughes MR, Graves ML, DeBruin EJ, Snyder K, Cipollone J, et al. Podocalyxin in the diagnosis and treatment of cancer. In: Mohan R, editor. Advances in cancer management. Rijeka, Croatia: InTech; 2012. p. 155-94.

23. Lacroix M, Leclercq G. Relevance of breast cancer cell lines as models for breast tumours: an update. Breast Cancer Res Treat. 2004;83:249-89.

24. Paxson JA, Gruntman A, Parkin CD, Mazan MR, Davis A, Ingenito EP, et al. Age-dependent decline in mouse lung regeneration with loss of lung fibroblast clonogenicity and increased myofibroblastic differentiation. PLoS One. 2011;6:e23232.

25. Babcook JS, Leslie KB, Olsen OA, Salmon RA, Schrader JW. A novel strategy for generating monoclonal antibodies from single, isolated lymphocytes producing antibodies of defined specificities. Proc Natl Acad Sci U S A 1996;93:7843-8.

26. Ao A, Morrison BJ, Wang H, López JA, Reynolds BA, Lu J. Response of estrogen receptor-positive breast cancer tumorspheres to antiestrogen treatments. PLoS One. 2011;6:e18810.

27. Grimshaw MJ, Cooper L, Papazisis K, Coleman JA, Bohnenkamp HR, Chiapero-Stanke L, et al. Mammosphere culture of metastatic breast cancer cells enriches for tumorigenic breast cancer cells. Breast Cancer Res. 2008; 10:R52

28. Ponti D, Costa A, Zaffaroni N, Pratesi G, Petrangolini G, Coradini D, et al. Isolation and in vitro propagation of tumorigenic breast cancer cells with stem/progenitor cell properties. Cancer Res. 2005;65:5506-11.

29. Dontu G, Abdallah WM, Foley JM, Jackson KW, Clarke MF, Kawamura MJ, et al. In vitro propagation and transcriptional profiling of human mammary stem/progenitor cells. Genes Dev. 2003;17:1253-70.

30. Fillmore CM, Kuperwasser C. Human breast cancer cell lines contain stemlike cells that self-renew, give rise to phenotypically diverse progeny and survive chemotherapy. Breast Cancer Res. 2008;10:R25.

31. Larsson A, Johansson ME, Wangefjord S, Gaber A, Nodin B, Kucharzewska P, et al. Overexpression of podocalyxin-like protein is an independent factor of poor prognosis in colorectal cancer. Br J Cancer. 2011;105:666-72.

32. Larsson AH, Nodin B, Syk I, Palmquist I, Uhlén M, Eberhard J, et al. Podocalyxin-like protein expression in primary colorectal cancer and synchronous lymph node metastases. Diagn Pathol. 2013;8:109.

33. Debruin EJ, Hughes MR, Sina C, Liu A, Cait J, Jian Z, et al. Podocalyxin regulates murine lung vascular permeability by altering endothelial cell adhesion. PLoS One. 2014;9:e108881.

34. Blanchet MR, Bennett JL, Gold MJ, Levantini E, Tenen DG, Girard M, et al. CD34 is required for dendritic cell trafficking and pathology in murine hypersensitivity pneumonitis. Am J Respir Crit Care Med. 2011;184:687-98.
35. Doyonnas R, Nielsen JS, Chelliah S, Drew E, Hara T, Miyajima A, et al. Podocalyxin is a CD34-related marker of murine hematopoietic stem cells and embryonic erythroid cells. Blood. 2005;105:4170-8.

36. Nielsen JS, McNagny KM. Novel functions of the CD34 family. J Cell Sci. 2008;121:3683-92.

37. Lin CW, Sun MS, Liao MY, Chung CH, Chi YH, Chiou LT, et al. Podocalyxin-like 1 promotes invadopodia formation and metastasis through activation of Rac1/ Cdc42/cortactin signaling in breast cancer cells. Carcinogenesis. 2014;35:2425-35.

\section{Submit your next manuscript to BioMed Central and take full advantage of:}

- Convenient online submission

- Thorough peer review

- No space constraints or color figure charges

- Immediate publication on acceptance

- Inclusion in PubMed, CAS, Scopus and Google Scholar

- Research which is freely available for redistribution 